\title{
Maps of the provincial distribution of Finnish Heteroptera
}

\author{
Tapio Lammes \& Veikko Rinne
}

Lammes, T. \& Rinne, V. 1990: Maps of the provincial distribution of Finnish Heteroptera. — Entomol. Fennica 1:209-220.

The distribution of Finnish Heteroptera (475 species) in biogeographical provinces is mapped, and their phenological pattern is presented, together with notes on the biology of some of the species. The most important faunistic literature on Finnish Heteroptera is listed.

Tapio Lammes, Sorolaisenkatu 6, SF-21200 Raisio, Finland

Veikko Rinne, Zoological Museum, University of Turku, SF-20500 Turku, Finland

The first checklist of the Finnish species of Heteroptera was published in 1935 by Lindberg, the latest in 1984 by Huldén \& Heikinheimo. Since the knowledge of the distribution of Heteroptera in Finland has increased enormously since Lindberg's days (e.g. Linnavuori 1966 and 1967), we consider the time ripe for an overview of the present situation.

We have been especially interested in the fauna of the northern parts of Finland and have made several collecting excursions there, partly together, partly separately. As a result we have been able to identify the northern distribution borders of many species.

The list is presented in the form of maps (pp. 214-220) and is based upon material in the collections of the Zoological Museum and the Department of Agricultural and Forest Zoology of the University of Helsinki, the Zoological Museum of the University of Turku, the entomological collections of Abo Akademi (Turku), the Kuopio Museum of Natural History, the Zoological Museum of the University of Oulu, and the private collections of $\mathrm{H}$. Luoma, I. Mannerkoski, V.-M. Mukkala, Y. Ranta, R. Roine, P. Turunen and, of course, our own collections. We have also had access to the undetermined material of the Zoological Museums, and have either determinined them or at least "sifted" through them as carefully as possible. The general survey of zoological collections was made before 1989 , but even the newest finds of many collectors during the summer of 1990 are included.

In addition to the publications mentioned in "References" we have also checked the meeting reports in all the more important Finnish scientific journals which deal with entomology. It follows, of course, that we have had to accept the correctness of the identifications presented. Only in a few clearly doubtful cases have we considered it better to exclude reports. Also "import species" have been excluded.

The species are mapped in alphabetical order. The nomenclature is principally in accordance with Huldén \& Heikinheimo (1984). We have, however, tried to include the latest nomenclatural changes.

In this work only species known from within the present borders of Finland are mapped. The small remaining parts of the biogeographical province LK (K1) have been joined to ES (Sa), No. 7. They constitute parts of the following municipalities: Uukuniemi, Saari, Parikkala and Rautjärvi (Simpele). We have also, according to general practice, divided both $\mathrm{PP}(\mathrm{Ob})$ and $\mathrm{KemL}$ (Lk) into two parts, OP (Obo) and PeP (Obu), 
and KiL (Lkk) and SoL (Lks), respectively. Thus the total number of provinces considered is twenty (Fig. 1). Half circles in the divided provinces, $\mathrm{OP}$ and PeP, and KiL and SoL, indicate that we do not know to which part a find belongs.

We have decided to include phenology in the maps, even if our knowledge about the period during which species can be found as imagines is far from complete. At least the rough division between species which hibernate as eggs or as imagoes can be deduced from the list of months beside the maps. The + sign at the beginning of the list represents January to April, and the + sign at the end September to December.

Species, for which there are notes on their biology, are indicated with an asterisk.

\section{Notes}

Atractotomus morio - Found living on Larix sp. Chlamydatus wilkinsoni - We have found it living in a moss carpet (Pleurozium schreberi) in dryish pine forests, where it seems to be rather common.

Criocoris crassicornis - See Oncotylus punctipes.

Dicyphus spp. - We have collected both of our two species exclusively on Melandrium rubrum.

Elatophilus nigricornis - We do not regard $E$. nigrellus separable from this species (see Pericart 1972).

Glaenocorisa propinqua - For the distribution of different subspecies, see Jansson (1986).

Monosynamma bohemani - In our opinion, only one Monosynamma species occurs in Finland. At present we consider it to be $M$. bohemani.

Oncotylus punctipes - In spite of the scarcity of the older material in Finnish collections, this species is amazingly abundant and widely distributed in SE Finland.

Orthocephalus vittipennis - The record of $O$. coriaceus (Lammes 1981) was based on a misidentification. The specimen is an exceptionally dark female of $O$. vittipennis. O. coriaceus must thus be excluded from the Finnish fauna.

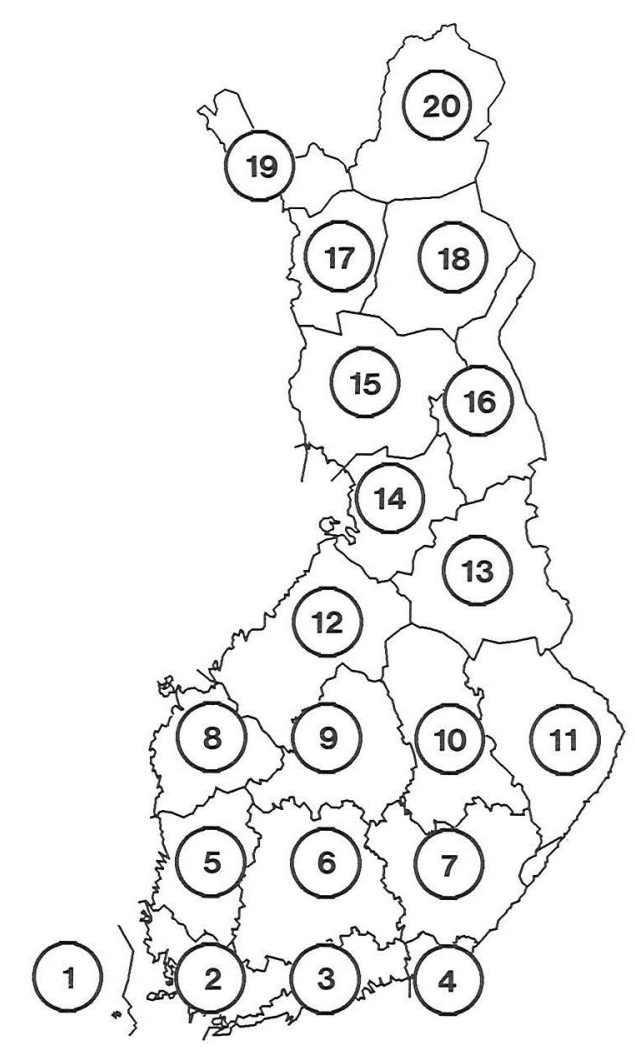

Fig. 1. The biogeographical provinces of Finland used in the maps. Within parentheses, abbreviations of the Finnish and Latin names are given, and the English name if it is not similar to the Finnish one. 1 = Ahvenanmaa $(A, A l), 2$ = Varsinais-Suomi $(V, A b), 3=$ Uusimaa $(\mathrm{U}, \mathrm{N}), 4$ = Etelä-Karjala (EK, Ka, South Karelia), 5 = Satakunta (St, St), 6 = Etelä-Häme $(E H$, Ta, South Häme), 7 = Etelä-Savo (ES, Sa, South Savo), 8 = Etelä-Pohjanmaa (EP, Oa, South Ostrobothnia), 9 = Pohjois-Häme (PH, Tb, North Häme), 10 = PohjoisSavo (PS, Sb, North Savo), 11 = Pohjois-Karjala (PK, $\mathrm{Kb}$, North Karelia), 12 = Keski-Pohjanmaa (KP, Om, Middle Ostrobothnia), $13=$ Kainuu (Kn, OK), 14 = Oulun Pohjanmaa (OP, Obo, North Ostrobothnia, $S$ part), 15 = Perä-Pohjanmaa (PeP, Obu, North Ostrobothnia, $\mathrm{N}$ part), $16=$ Koillismaa (Ks, Ks), $17=$ Kittilän Lappi (KiL, Lkk, Kemi Lapland, W part), 18 = Sompion Lappi (SoL, Lks, Kemi Lapland, E part), 19 = Enontekiön Lappi (EnL, Le, Enontekiö Lapland), and 20 = Inarin Lappi (InL, Li, Inari Lapland).

Pithanus hrabei - The northernmost material of the genus Pithanus seems to be exclusively P. hrabei. 
Pityopsallus lapponicus - The host plants of this species in Finland are Salix spp. (e.g. S. phylicifolia, S. glauca, S. lapponum).

Psallus scholtzi - At present we have included earlier records of $P$. alnicola (Kerzhner \& Yachewskii 1967).

Stalia boops - The species has been overlooked because of its cryptic way of living. It can be found in humid to moist meadows at the ground level. The species is possibly nocturnal.

Teratocoris saundersi - The species has two distinct southern and northern populations in Finland, which are morphologically different and do not overlap geographically. The subspecies described by Wagner (1965) are not, however, included, because in our opinion this case needs to be studied more.

Xylocoris cursitans - The species was erroneously reported from InL (Lammes 1979).

Acknowledgements. We are sincerely grateful to the staff of the Entomological Museums listed above for their generous help. We also thank the private collectors mentioned earlier in this paper. We are deeply grateful to Dr. Rauno Linnavuori, whose excellent work on Finnish bugs, "Luteet 1-2", introduced us to this fascinating group.

\section{References and other faunistic literature}

Albrecht, A. 1977: Intressanta fynd av skinnbaggar och stritar i Finland (Heteroptera \& Homoptera, Auchenorrhyncha). - Notulae Entomol. 57:51-52.

Albrecht, A., Huldén, L., Lammes, T. \& Rinne, V. 1984: Heteroptera of Inari Lapland. - Kevo Notes 7:41-46.

Bagge, P. 1982: Caddis flies (Trichoptera) and water bugs (Heteroptera, Corixidae) of small water bodies caught by light trapping in southeastern Finland). - Notulae Entomol. 62:73-81.

- 1983: The macrobenthos of the River Tourujoki and its tributaries (Central Finland). 2. Odonata, Heteroptera and Coleoptera. - Acta Entomol. Fennica 42: $15-22$.

Biström, O. 1978: Coleoptera and Heteroptera in a natural forest in Mäntyharju (Southern Finland). — Notulae Entomol. 58:95-100.

Biström, O. \& Väisänen, R. 1988: Ancient-forest invertebrates of the Pyhän-Häkki national park in Central Finland. - Acta Zool. Fennica 185:1-69.

Brander, T. \& Huldén, L. 1971: Lounais-Hämeen luteista, Heteroptera. — Lounais-Hämeen Luonto 43:9-15.

Coulianos, C.-C. \& Ossiannilsson, F. 1976: Catalogus insectorum Sueciae. VII. Hemiptera-Heteroptera. 2nd ed. - Entomol. Tidskr. 97:135-173.
Frey, R. 1916-1917: Entomologiska anteckningar från norra Savolaks. - Meddel. Soc. Fauna Flora Fennica 43: 84-97.

Gaun, S. 1974: Blomstertaeger. Danmarks Fauna. 81. København. 279 pp.

Gyllensvärd, N. 1964: A key to Swedish Aradidae (Hem. Het.) with figures of the male genitalia. - Opuscula Entomol. 29(1-2):110-116.

Heliövaara, K. \& Väisänen, R. 1983: Environmental changes and the flat bugs (Heteroptera, Aradidae and Aneuridae). Distribution and abundance in Eastern Fennoscandia. - Ann. Entomol. Fennici 49:103-109.

Hellén, W. 1926: Hemipterologische Notizen aus Finnland. - Notulae Entomol. 6:9-15.

- 1932: Hemiptera från Enare Lappmark. — Notulae Entomol. 12:58-59.

- 1976: Verzeichnis der in der Jahren 1971-75 für die Fauna Finnlands neuhinzugekommenen Insektenarten. - Notulae Entomol. 56:109-120.

Hirvenoja, M. 1964: Studien über die Wasserinsekten in Riihimäki (Südfinnland). IV. Ephemeroptera, Odonata, Hemiptera, Lepidoptera und Coleoptera. - Ann. Entomol. Fennici 30(2):65-93.

Huldén, L. 1975: Faunistic notes on Hemiptera from Finland I. - Notulae Entomol. 55:86-88.

- 1980: Nabis punctatus Costa (Nabidae) ny för Finland (EFH/HHY 1980-03-19). — Notulae Entomol. 60:227.

- 1982: Microvelia umbricola Wrobl. (Microveliidae) ny för Finland (EFH/HHY 1981-09-23). — Notulae Entomol. 62:153.

- 1985: Kommentarer till den nya hemipterkatalogen. - Notulae Entomol. 65:160.

- 1985: Pachytomella parallela (Meyer-Dür) (Miridae) ny för Finland. - Notulae Entomol. 65:160.

Huldén, L. \& Heikinheimo, O. 1984: Checklist of Finnish insects. Hemipteroidea. - Notulae Entomol. 64: 97-124.

Huldén, L., Meinander, M., Nybom, O. \& Silfverberg, H. 1977: Deletions from the Finnish Fauna I. - Notulae Entomol. 57:11-12.

Jansson, A. 1965: Three species of Corixidae (Hem.Het.) new to Finland. - Ann. Entomol. Fennici 31:75-76.

- 1969: Identification of larval Corixidae (Heteroptera) of Northern Europe. - Ann. Zool. Fennici 6:289-312.

- 1976: Records on the distribution of Micronecta species (Het., Corixidae) in Finland. - Ann. Entomol. Fennici 42:162-166.

- 1982: Faunistic notes on some Fennoscandian Corixidae (Heteroptera). - Notulae Entomol. 62:143-144.

- 1996: The Corixidae (Heteroptera) of Europe and some adjacent regions. - Acta Entomol. Fennica 47:1-94.

Kaisila, J. 1952: Insects from arctic mountain snows. Arn. Entomol. Fennici 18:8-25.

Kerzhrer, I. M. \& Yachewskii, T. L. 1967: (1964 in Russian) Order Hemiptera (Heteroptera). - In: BeiBienko, G. Ya. (ed.), Keys to the fauna of USSR 84:851-1118. Israel Progr. Sci. Transl., Jerusalem. 
Krogerus, H. 1932: Über die Ökologie und Verbreitung der Arthropoden der Triebsandgebiete an den Küsten Finnlands. - Acta Zool. Fennica 12:1-308.

Lammes, T. 1979: Pohjoisia, maakunnilleen uusia ludelöytöjä (TEKS 1979-04-25). — Notulae Entomol. 59:168.

- 1981: Orthocephalus coriaceus (Heteroptera, Miridae) found in Finland. - Notulae Entomol. 61:130.

- 1982: Maalle uusi ludelaji Oncotylus punctipes Reut. (Miridae) (EFH/HHY 1982-02-17). — Notulae Entomol. 62:153.

- 1984: Maalle uusi ludelaji Chorosoma schillingi (Schumm.) (Rhopalidae) (TEKS 1983-11-16). — Notulae Entomol. 64:197.

- 1987: Trigonotylus fuscitarsis sp.n. (Heteroptera, Miridae) from northern Europe. - Ann. Entomol. Fennici 53:69-71.

Lindberg, H. 1921: Hemiptera Heteroptera på Lojo ås. Notulae Entomol. 1:19-20.

- 1921: Nya fyndorter för sällsynta stinkflyn. - Notulae Entomol. 1:116-117.

- 1921: Ålands Hemiptera Heteroptera. - Medd. Soc. Fauna Flora Fennica 46:32.

- 1923: Anteckningar om fynd av stinkflyn i Finland. Notulae Entomol. 3:88.

- 1924: Zur Kenntnis der Corixiden Ostfennoskandiens. - Acta Soc. Fauna Flora Fennica 56(2):1-6.

- 1927: Zur Kenntnis der subalpinen und alpinen Hemipterenfauna von Enontekis-Lappland. - Memoranda Soc. Fauna Flora Fennica 1:63-68.

- 1930: Insektenfunde auf einen Schäreninsel am äussersten Küstensaum von Süd-Finnland. - Notulae Entomol. 10:122-123.

- 1935: Hemiptera. Heteroptera. - Enumeratio Insectorum Fenniae 3:1-7.

- 1935: Uppgifter angående några hemipterer från Finland. - Notulae Entomol. 15:62-63.

- 1937: Ökologische Studien über die Coleopteren- und Hemipterenfauna im Meere in der Pojo-Wiek und im Schärenarchipel von Ekenäs in Südfinnland. - Acta Soc. Fauna Flora Fennica 60:516-572.

- 1940: Antekningar om skalbaggs- och skinnbaggsarter på hedmarker på Hangö udd. - Memoranda Soc. Fauna Flora Fennica 17:3-6.

- 1941-1942: Hemipterfynd i provinserna Oa och Om år 1940. - Memoranda Soc. Fauna Flora Fennica 18:21-24.

- 1944: Ökologisch-geographische Untersuchungen zur Insektenfauna der Felsentümpel an den Küsten Finnlands. - Acta Zool. Fennica 41:1-178.

- 1950: Om några hemipterer, som under senaste tid invandrat till Finland. - Notulae Entomol. 30:49-54.

- 1951: Über ostfennoskandische Funde dreier Callicorixa-Arten. - Notulae Entomol. 31:127-128.

- 1955: Uppgifter om nya mirider för Finlands fauna. Notulae Entomol. 35:63.

- 1957: Megaloceraea recticornis Geoffr., en för Finland ny skinnbagge. - Notulae Entomol. 37:126.
- 1963: Notonecta reuteri Hung. - Notulae Entomol. 43:131-132.

Lindberg, H. \& Saris, N.-E. 1952: Insektfaunan i Pisavaara naturpark (Finland, prov. Ob). - Acta Soc. Fauna Flora Fennica 69(2):1-82.

Lindskog, P. 1974: Distributional and systematic notes on Saldula fucicola (J.Sahlb.) and some other shore bugs of Eastern Fennoscandia (Heteroptera, Saldidae). Notulae Entomol. 54(2):33-56.

Linnavuori, R. 1949: Havaintoja Etelä-Hämeen lude- ja kaskaseläimistöstä. - Ann. Entomol. Fennici 15(2):63-71.

- 1950: Lisähavaintoja Etelä-Hämeen nivelkärsäiseläimistöstä. — Ann. Entomol. Fennici 16(3):121-125.

- 1950: Tutkimuksia Suomen vesilude-eläimistöstä. Ann. Entomol. Fennici 16(2):63-71.

- 1951: Hemipterological observations. - Ann. Entomol. Fennici 17:51-65.

- 1951: Investigations on the species of the genus Micracanthia Reut. (Het., Saldidae). - Ann. Entomol. Fennici 17(4):154-158.

- 1951: Studies on the family Cryptostemmatidae. Ann. Entomol. Fennici 17(3):92-103.

- 1952: Havaintoja etelä- ja pohjois-Savon nivelkärsäiseläimistöstä. - Ann. Entomol. Fennici 18(2):64-75.

- 1961: On some Finnish Heteroptera. - Ann. Entomol. Fennici 27(1):53-55.

- 1966: Nivelkärsäiset I. Luteet 1. Animalia Fennica 10. - Helsinki. 205 pp.

- 1967: Nivelkärsäiset II. Luteet 2. Animalia Fennica 11. - Helsinki. 232 pp.

Meinander, M. 1972: The invertebrate fauna of the Kilpisjärvi area, Finnish Lapland. 7. Hemiptera. - Acta Soc. Fauna Flora Fennica 80:67-92.

Ossiannilsson, F. 1967: On the lectotypus of Psallus lapponicus Reuter and Psallus piceae Reuter (Hem., Heteropt.). — Entomol. Tidskr. 88:87-88.

Pajunen, V. I. \& Jansson, A. 1969: Dispersal of the rock pool corixids Arctocorisa carinata (Sahlb.) and Callicorixa producta (Reut.) (Heteroptera, Corixidae). Ann. Zool. Fennici 6:391-427.

Pericart, J. 1972: Hémiptères Anthocoridae, Cimicidae et Microphysidae de l'ouest-paléarctique. - Paris. 402 pp.

Perttula, T. 1984: Annotated list of bugs (Hemiptera-Heteroptera) from the coastal sand dunes in the vicinity of Tvärminne Zoological Station, Finland. - Memoranda Soc. Fauna Flora Fennica 60:23-26.

Poppius, B. 1909: Über die Verbreitung einiger an der Meeres-Küsten lebenden Coleopteren und Hemipteren in Fennoscandia. - Medd. Soc. Fauna Flora Fennica 35:59-62.

Reuter, O. M. 1871: Pargas sockens Heteroptera. - Notiser Sällsk. Fauna Flora Fennica Förhandl. 11:309-326.

- 1872: Skandinaviens och Finlands Aradider. - Öfv. Kongl. Vet.-Acad. Förhandl. 29(5):47-62.

- 1875: Hemiptera Gymnocerata Scandinaviae et Fen- 
niae. I. Cimicidae (Capsina). - Acta Soc. Fauna Flora Fennica 1(1):1-206.

- 1878: Actinocoris, novum Hemipterorum genus e Fennia australi. - Medd. Soc. Fauna Flora Fennica 2:194-197.

- 1880: Finlands och den Skandinaviska halföns Heteroptera. - Entomol. Tidskr. 1:113-145.

— 1880: Nya bidrag till Åbo och Ålands skärgårds Hemipter-fauna. - Medd. Soc. Fauna Flora Fennica 5:160-236.

- 1881: En ny art Aradus från Lappland. — Medd. Soc. Fauna Flora Fennica 7:139-143.

- 1881: Finlands och den Skandinaviska halföns Hemiptera - Heteroptera (forts.). — Entomol. Tidskr. 2: 61-93.

- 1882: Finlands och den Skandinaviska halföns Hemiptera - Heteroptera (forts.). - Entomol. Tidskr. 3:65-81, 105-121, 163-172.

- 1884: Finlands och den Skandinaviska halföns Hemiptera - Heteroptera (forts.). — Entomol. Tidskr. 5:173-185.

- 1891: De skandinaviskt finska Acanthia- (Salda-) arterna of saltatoria -gruppen. - Medd. Soc. Fauna Flora Fennica 17:144-160

- 1900: De finska arterna af Aradus lugubris -gruppen. — Medd. Soc. Fauna Flora Fennica 26:131-139.

- 1913: Vägglusfamiljen (Cimicidae), des fylogeni, systematik, ekologi och utbredning. - Entomol. Tidskr. 34:1-21.

Rinne, V. 1979: Ludelöytöjä (Miridae) Varsinais-Suomesta (TEKS 1978-10-18). — Notulae Entomol. 59:168.

- 1979: Suomelle uusi ludelaji (Miridae) (TEKS 197811-15). — Notulae Entomol. 59:168.

- 1982: Suomelle uusi ludelaji Campylomma verbasci (M.-D.) (Miridae) (TEKS 1981-03-18). — Notulae Entomol. 62:153.

- 1984: Uusia ludelöytöjä Suomesta (Heteroptera). Notulae Entomol. 64:88-89.

- 1989: Review of the European Polymerus subgenus Poeciloscytus (Heteroptera, Miridae), with two new species and special reference to the Finnish fauna. Ann. Entomol. Fennici 55:89-101.

Sahlberg, J. 1867: Entomologiska anteckningar från en resa i sydöstra Karelen sommaren 1866. I. Orthoptera och Hemiptera. - Notiser Sällsk. Fauna Flora Fennica Förhandl. 9:159-197.

- 1867: Hemiptera samlade i Torneå Lappmark år 1867. — Notiser Sällsk. Fauna Flora Fennica Förhandl. 9:223.

- 1875: Synopsis Amphibicorisarum et Hydrocorisarum Fenniae. - Notiser Sällsk. Fauna Flora Fennica Förhandl. 14:241-301.

- 1881: Enumeratio Hemipterorum Gymnoceratorum Fenniae. - Medd. Soc. Fauna Flora Fennica 7:1-109.

- 1920: Enumeratio Hemipterorum Heteropterorum Faunae Fennicae. Editio secunda aucta et emendata. — Bidrag Kännedom Finlands Natur Folk 79(2):1-227.

Sahlberg, R. F. 1848: Monographia Geocorisarum Fenniae. - xi+155 pp. Helsinki.

Söderman, G. 1970: Myrmecophyes alboornatus Stål ny för Finland (Heteroptera, Miridae). — Notulae Entomol. 50(3): 96 .

Stichel, W. 1955-62: Illustrierte Bestimmungstabellen der Wanzen. II:1-4 (Europa). - Berlin.

Tiensuu, L. 1965: Stictopleurus punctatonervosus Goeze, Suomelle uusi lude. - Kymenlaakson Luonto 6(2):14.

Valkeila, E. 1977: Euryopicoris nitidus (Heteroptera, Miridae) löydetty Suomesta. - Notulae Entomol. 57:59-60.

Vepsäläinen, K. 1973: Gerris sphagnetorum Gaunitz (Heteroptera, Gerridae) in Finland. - Notulae Entomol. 53:59-60.

- 1973: The distribution and habitats of Gerris Fabr. species (Heteroptera, Gerridae) in Finland. - Ann. Zool. Fennici 10:419-444.

Vikberg, V. 1978: Euryopicoris nitidus (M.-D.) (Miridae) löydetty Suomesta (EFH/HHY + SHS 1976-10-15). — Notulae Entomol. 58:172.

von Pfaler, E. 1936: Lebenszyklen der Lygaeiden (Hem.). - Notulae Entomol. 16:65-85.

Wagner, E. 1954: Euryopicoris fennicus nov. spec., eine neue Miriden-Art aus Finnland (Hemipt. Heteropt.). — Notulae Entomol. 34:82-84.

- 1955: Lyctocoris nidicola n.sp., eine neue Anthocoriden-Art aus Finnland (Hem. Het.). - Notulae Entomol. 35:60-62.

- 1965: Über Teratoccris Fieber 1858 (Heteroptera, Miridae). — Notulae Entomol. 45:138-144.

Received 4.I.1990 


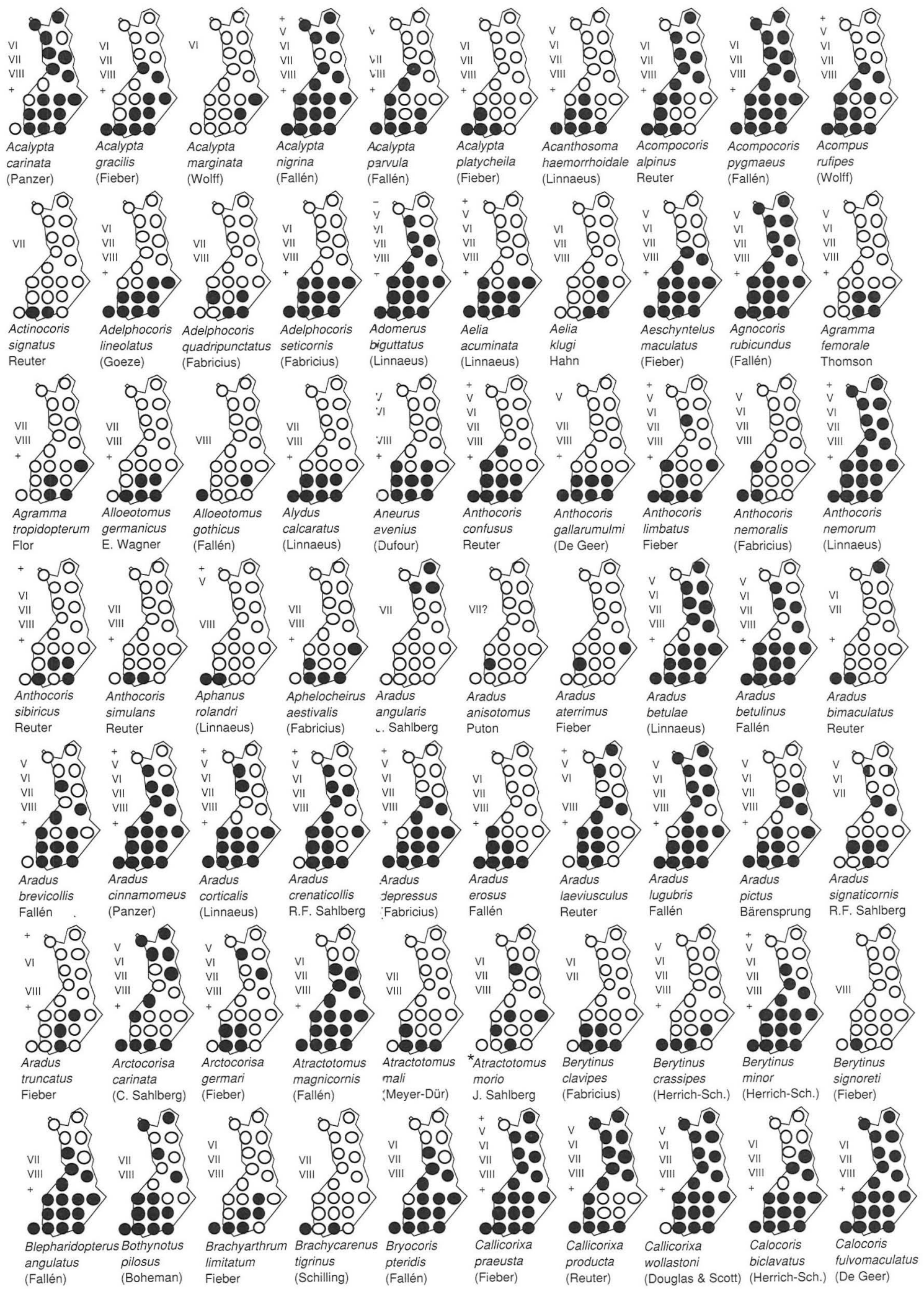




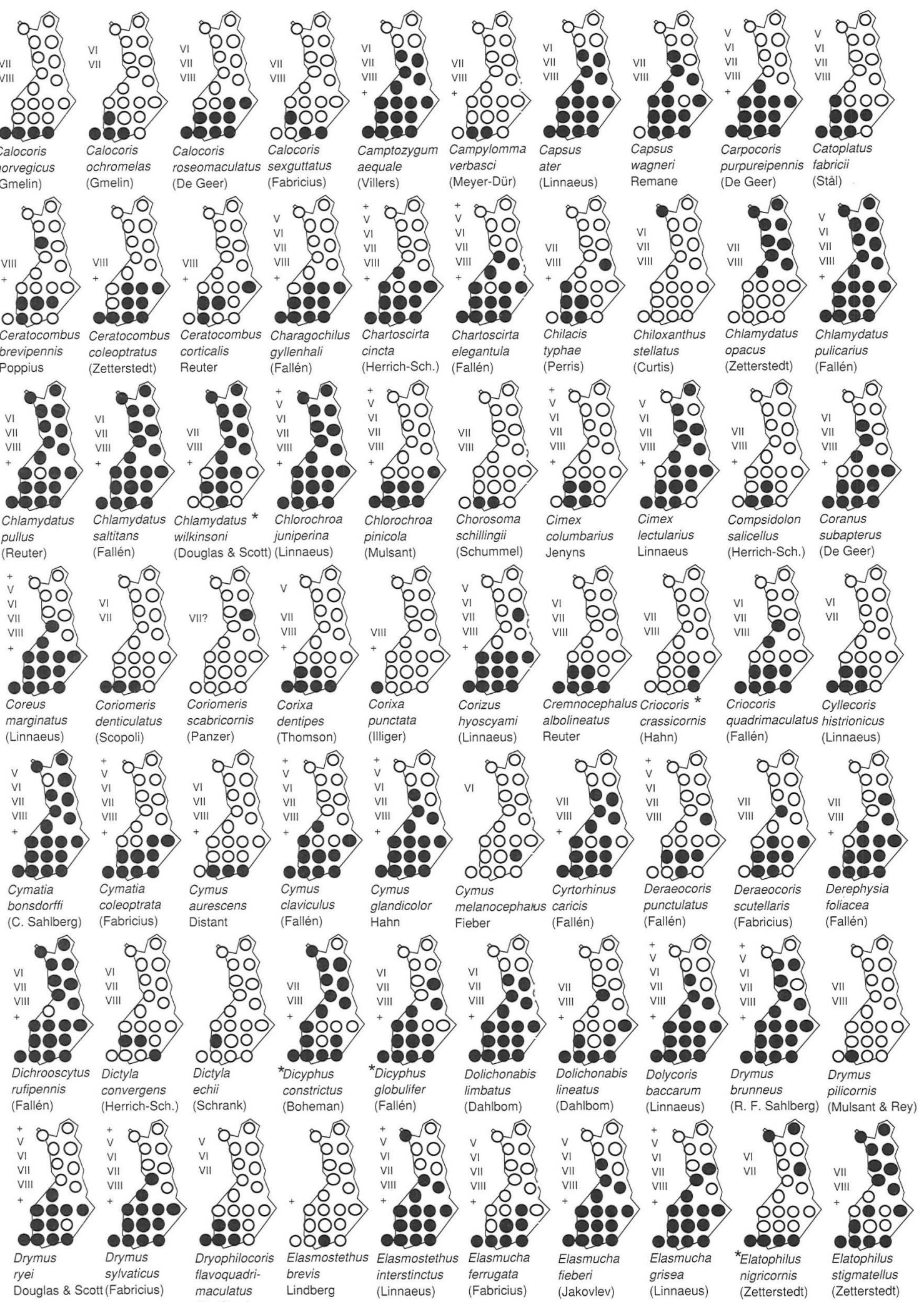




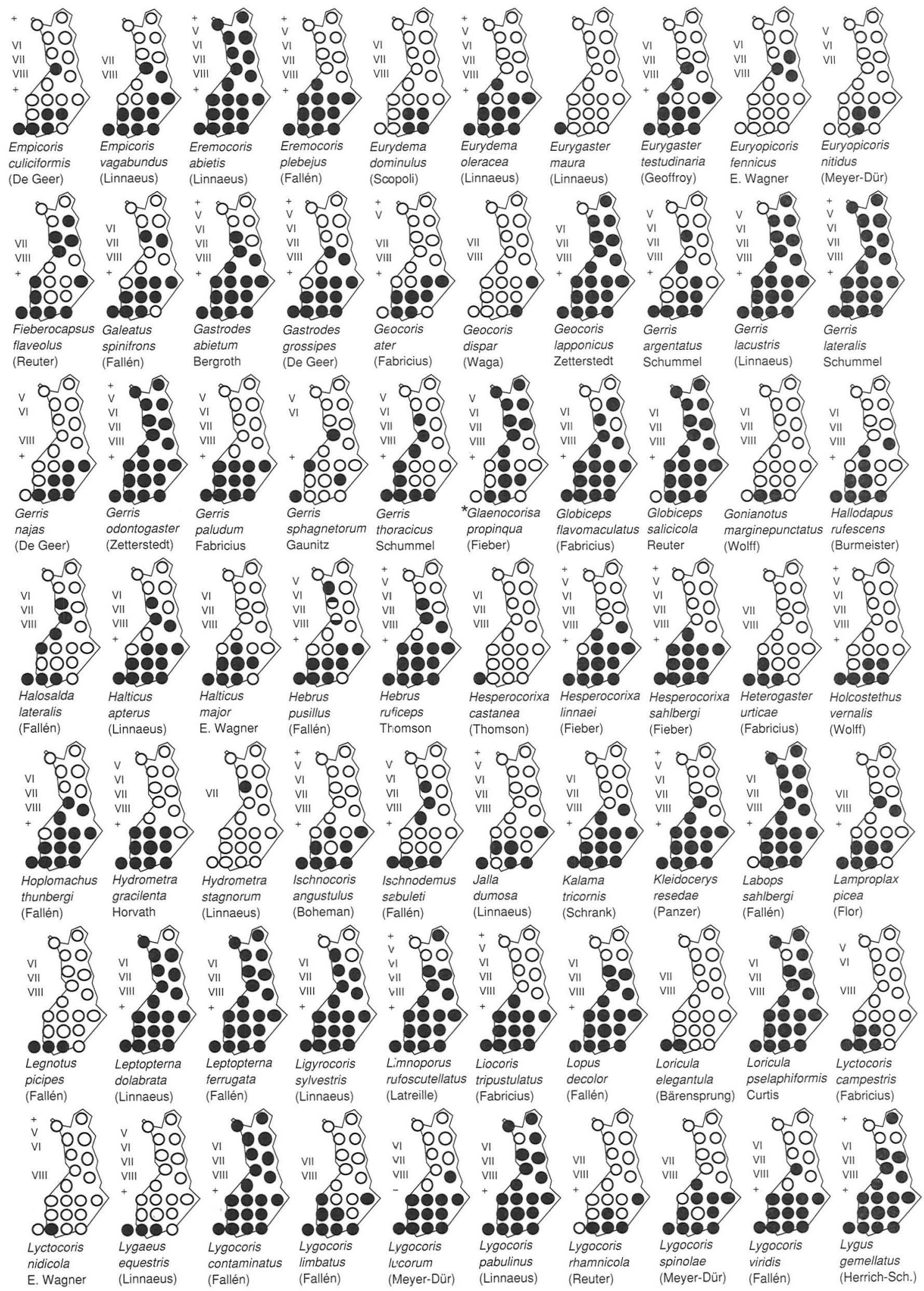




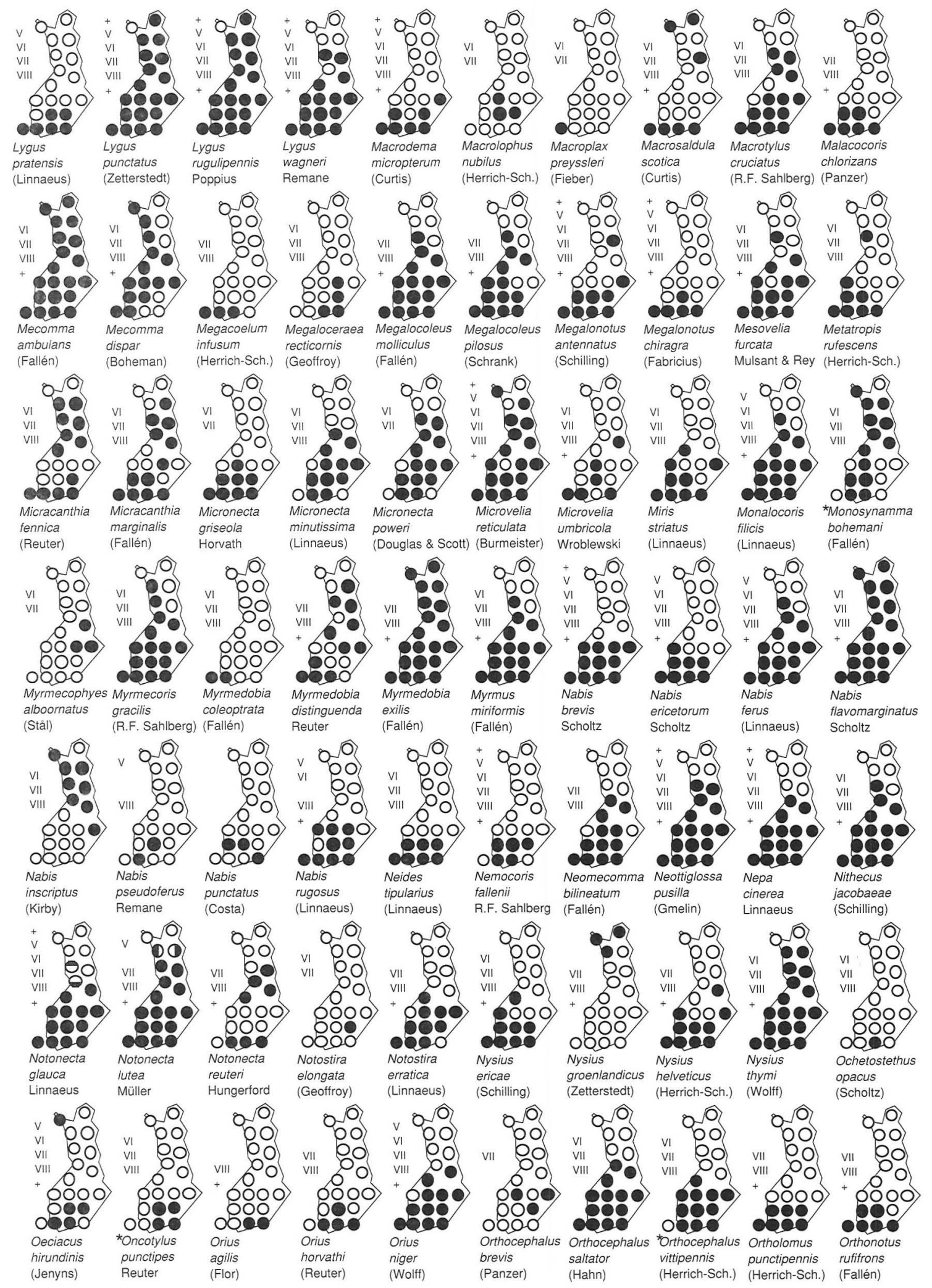




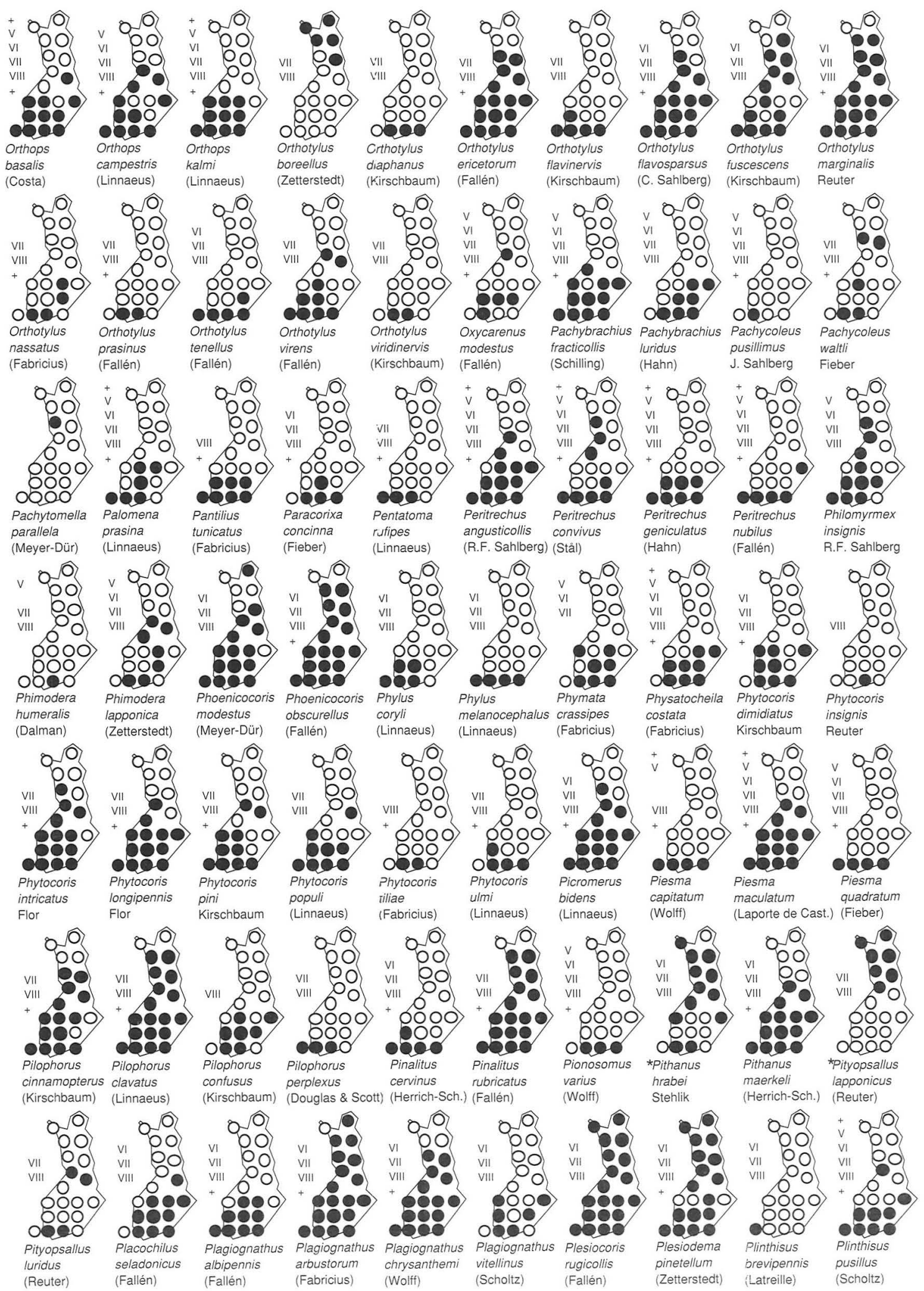




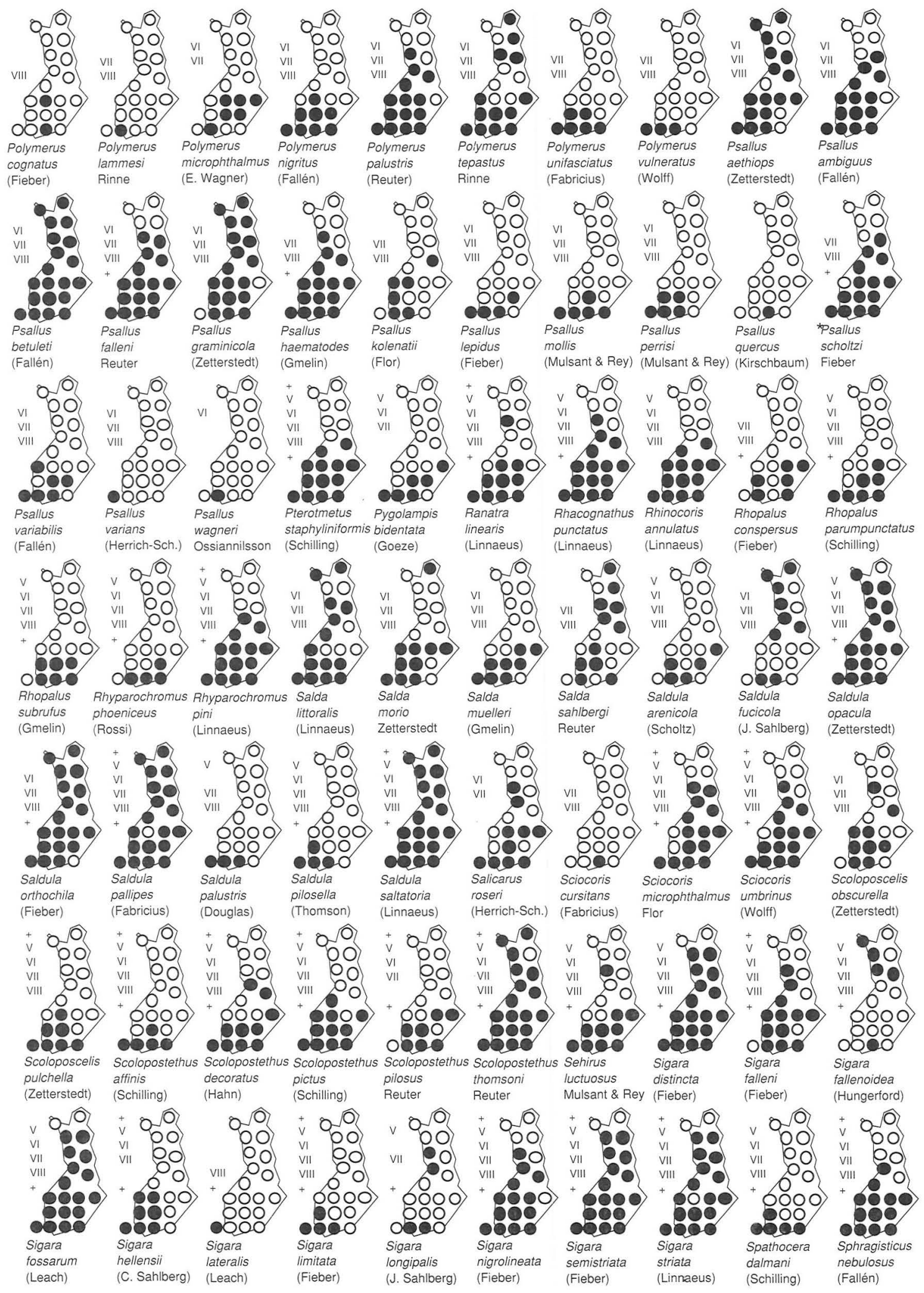



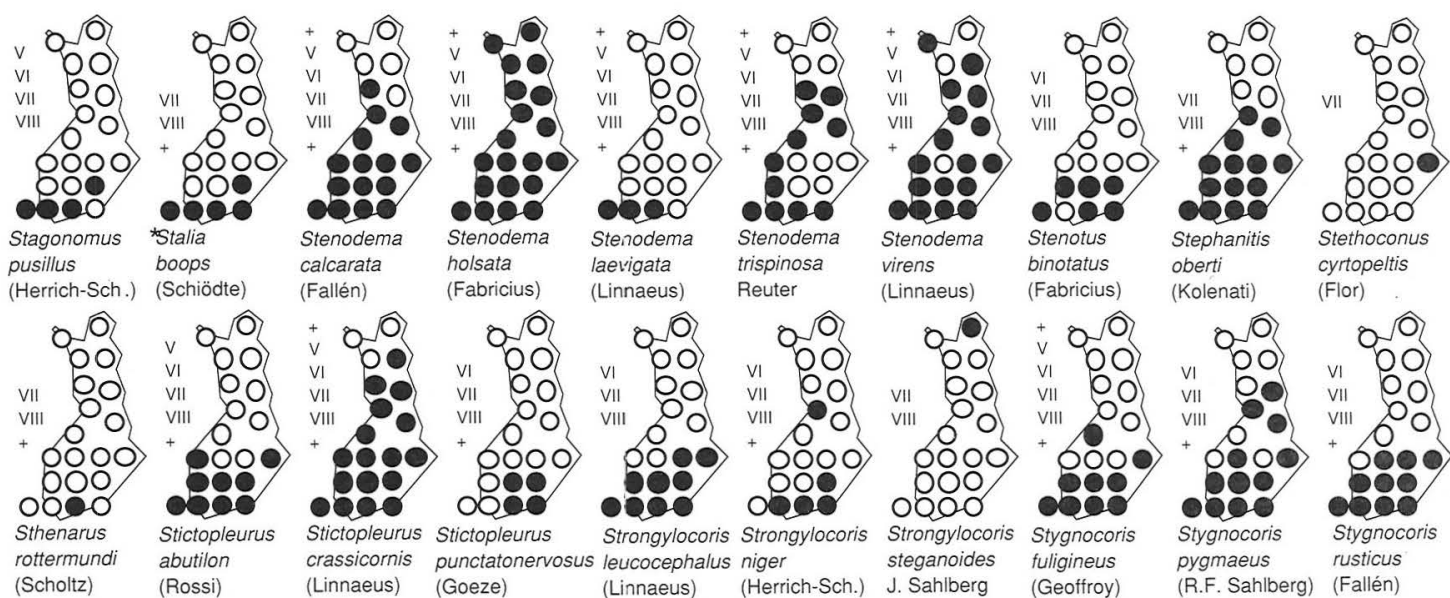

¿ 0 -

(Fabricius)

laevigata

OO

0

o 0

o

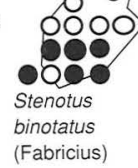

(Kolenati)

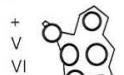

o

ô
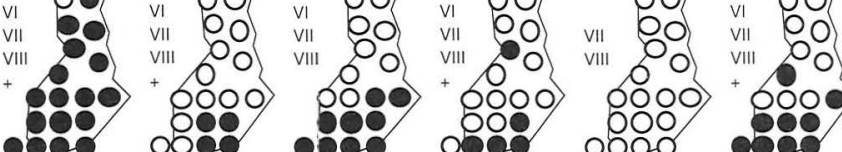

000 - ar 000
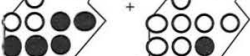

Stictopleurus Stictopleurus

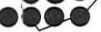

\section{o8e.}

0000 잉

crassicornis punctatonervosus leucocephalus niger
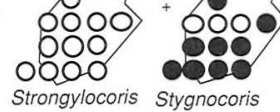

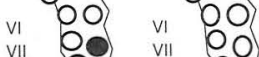

(Scholtz) (Rossi)

(Linnaeus)

(Herrich-Sch.)

teganoides fuligineus

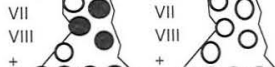

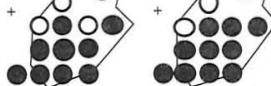

Stygnocoris

Stygnocoris

pygmaeus

rusticus

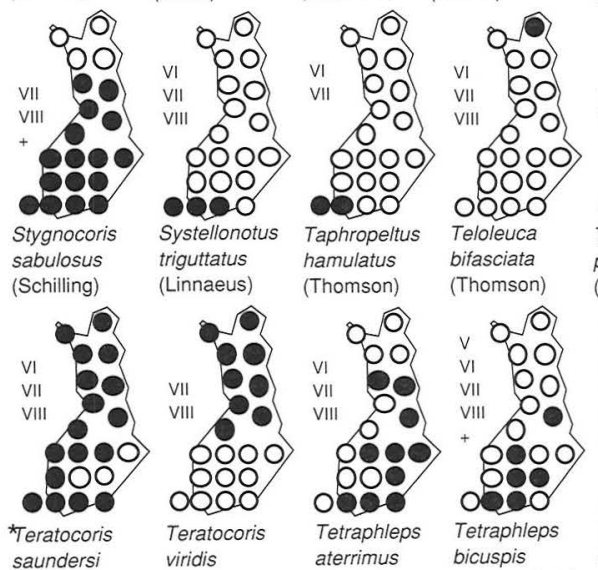

20

II

vero 0200

Teileuca

pellucens

(Fabricius)

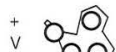

vi vil 60

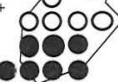

scarabaeoides cardui

Oo
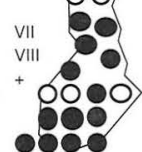

.

gracilis

(Horvath)

0

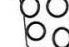

0 O?

O
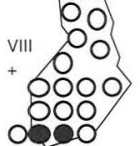

pusillus

v1 00
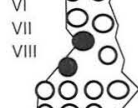

\section{Q0}

\section{Teratocoris}

(Herrich-Sch.) (Boheman)

i OO

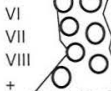

00
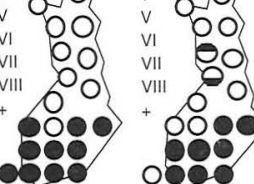

saundersi

viridis

aterrimus

bicuspis
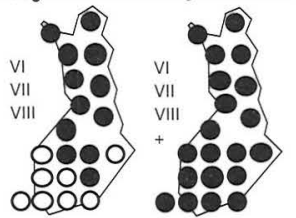

Oे

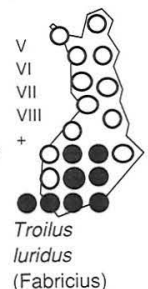

$+200$

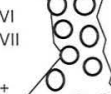

$+6000$

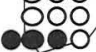

Trigonotylus

fuscitarsis

Lammes

Trigonotylus

Tritomegas

bicolor

(Geoffroy)

(Linnaeus)

(Fabricius)
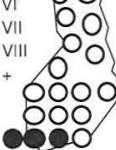

\section{Tropistethus}

halosericeus

(Ssholtz)

(Linnaeus)

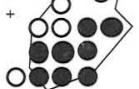

Trapezonotus

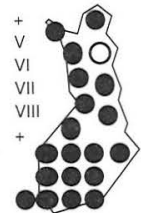

anorus

Trapezonotus

arenarius

(Flor)

(Linnaeus)

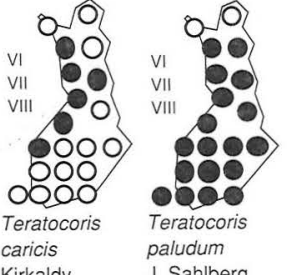

Kirkaldy

J. Sahlberg

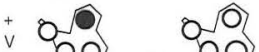

$\checkmark$ o) $v 100$
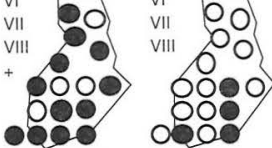

Trapezonotus Trigonotylus

desertus coelestialium
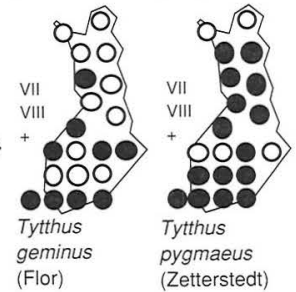

$+0 \widehat{0}$

$\begin{array}{lll}1 & 0 \\ v & 0 & 0\end{array}$
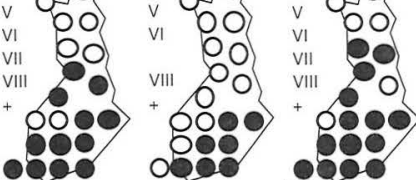

Velia

saulii

Ulmicola

pinipes

Tamanin

(Flor)<smiles></smiles><smiles>c1ccoc1</smiles>

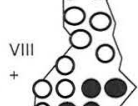

vil 80

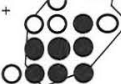

xylocoris

formicetorum vII $\mathrm{OO}$ vili 60 080

xylocoris

galactinus

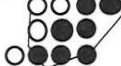

xylocoris

lativentris

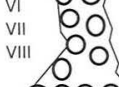

$v 0$
$v 0$

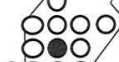

.280

xylocoris

(J. Sahlberg) (Reuter)

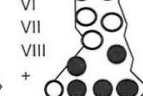

caerulea

(Linnaeus) 